\title{
Fluxômetro hidrodinâmico em cirurgia de revascularização do miocárdio
}

\author{
Fábio Biscegli JATENE*, Pedro C. P. LEMOS*, Adib D. JATENE*, São Paulo, SP.
}

RBCCV

\begin{abstract}
JATENE, F. B.; LEMOS P. C. P.; JATENE, A. D. - Fluxômetro hidrodinâmico em cirurgia de revascularização do miocárdio. Rev. Bras. Cir. Cardiovasc., 1 (1): 49-53, 1986.

RESUMO: A avaliaçāo do fluxo pelas pontes de veia safena na cirurgia de revascularização do miocárdio fornece uma informação segura do aporte sangüíneo ao miocárdio, bem como da perspectiva de perviabilidade destas anastomoses a longo prazo. Contudo, em nosso meio, a disponibilidade de fluxômetros eletrónicos, habitualmente utilizados, é, por vezes, difícil, sobretudo em função de seu custo e manutenção. O sistema desenvolvido consta de um conector para a linha arterial, com saída lateral, ligado a um tubo de $Y$. Uma das extremidades é conectada a um manómetro e a outra, à veia safena, ao término da anastomose distal. Como a saída lateral tem diâmetro conhecido, a queda da pressão, quando se liga o sistema para a veia safena, é proporcional ao fluxo. Uma tabela obtida por calibração prévia, permite conhecer, instantaneamente, o fluxo para a artéria tratada. Outra vantagem do sistema é a irrigação seletiva para a árèa tratada antes da realizaçăo da anastomose proximal, situação favorável quando existem áreas severamente isquêmicas. Este sistema, em uso corrente em nosso Serviço, vem provando a sua eficiência, aliada à sua praticidade e ao custo irrelevante.
\end{abstract}

DESCRITORES: fluxômetro hidrodinâmico; miocárdio, revascularização.

\section{INTRODUÇÃO}

Oestudo do fluxo pelas anastomoses aorto-coronárias, com segmentos de veia safena, é de real valor na cirurgia de revascularizaçāo do miocárdio. sobretudo para a avaliação do desempenho das anastomoses a curto e longo prazo. É praticamente obrigatória a revisão de uma anastomose que tenha apresentado fluxo inexplicavelmente muito reduzido, ao fim de sua realização. Com relação à avaliação destes fluxos, a magnitude, ou seja, a avaliação quantitativa que é representada pelo fluxo médio dos enxertos, ocupa lugar de destaque. Entretanto, outras características do fluxo parecem de- sempenhar, também, o seu papel na evolução satisfatória destes enxertos. Entre estas, estão a relação entre os componentes sistólicos e diastólicos, além das variaçōes nas velocidades do fluxo ${ }^{1,4,9}$.

Contudo, sobretudo em nosso meio, a avaliação do fluxo não é usualmente realizada, devido, tanto a fatores técnicos, quanto a dificuldades no manejo e na manutenção destes aparelhos.

Tendo em vista estes aspectos, os autores propõem, no presente estudo, um dispositivo simples, prático e de custo irrelevante, para realizar medidas do fluxo sangüíneo nas pontes de veia safena.

\footnotetext{
Trabalho realizado no Instituto do Coraçāo do Hospital das Clinicas da Faculdade de Medicina da Universidade de São Paulo. Săo Paulo, SP. Brasil. 


\section{MATERIAL E METODO}

O sistema é montado previamente à instalação da circulação extracorpórea, utilizando-se tubo plástico em $Y$ de equipo de infusão de soluções parenterais e medida de pressão venosa.

Entre a cânula da aorta e a linha arterial, interpõe-se um conector metálico de $3 / 8$ ", que possui um pequeno ramo lateral com diâmetro interno de $1 \mathrm{~mm}$, ao qual é acoplado um dos ramos do equipo plástico em $\mathrm{Y}$. O segundo ramo do equipo é mantido fechado, no campo operatório, e o terceiro ra$\mathrm{mo}$, fora do campo operatório, é acoplado a um manômetro de mercúrio. Este ramo permanece aberto e em constante funcionamento durante a fase de circulação extracorpórea (Figura 1). O sistema é totalmente preenchido por sangue heparinizado vindo diretamente da linha arterial.

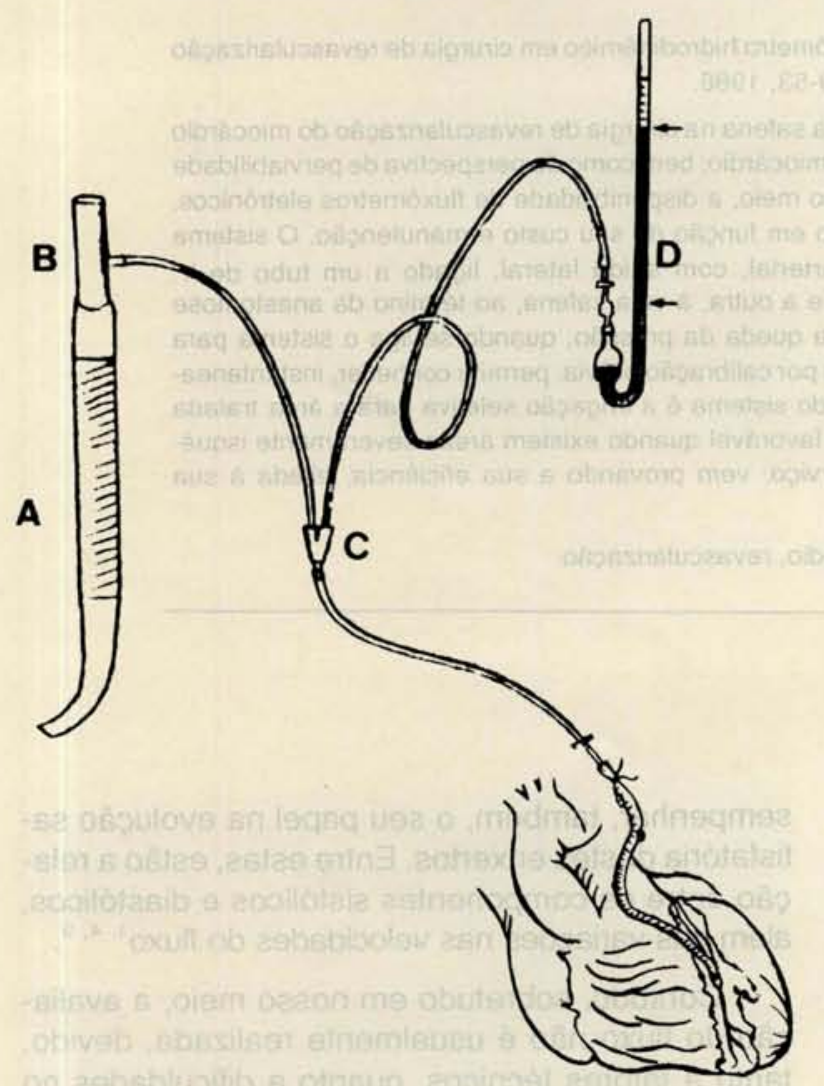

Fig. 1 - Esquema do disposto proposto

A - Canula arterial

B - Conector com saida lateral

C - Conexảo em $Y$

D - Manometria de mercúrio

Após o término da anastomose safeno-coronária, acopla-se o ramo do equipo, que permanece ainda fechado no campo operatório, à extremidade livre da veia safena. Neste momento, é observada a pressão registrada no manômetro, a qual é a pressão média da linha arterial. A seguir, abre-se a pinça do ramo do equipo ligado à veia, permitin- do-se, então, que o sangue vindo da linha arterial se dirija à veia safena e, daí, até a artéria coronária. Neste momento, observa-se uma queda da pressão no manômetro. A diferença entre as pressōes registradas, prévia e posteriormente à abertura da pinça, corresponde ao fluxo de sangue que se dirigiu à coronária.

\section{RESULTADOS}

Para a obtenção dos valores médios de fluxo sangüíneo em função das variações de pressão, o sistema foi, inicialmente, calibrado in vitro. Nestas condiçōes, foram avaliados valores de pressão no dispositivo, prévia e posteriormente à abertura do ramo dirigido ao campo operatório, com os correspondentes fluxos em milimetros por minuto ( $\mathrm{ml} /$ $\mathrm{min})$. Estas medidas foram repetidas para todos os valores possíveis de variação pressórica, do valor mínimo ao máximo; com fluxo correspondente a cada valor. O hematócrito, durante esta avaliação, foi mantido em $28 \%$. Todos os valores obtidos estâo representados na Tabela 1.

\section{TABELA 1}

Demonstrativo dos fluxos em ml/min, obtidos em funçâo da pressão inicial na linha arterial em $\mathrm{mmHg}(P I)$ e dos valores da variaçáo pressórica em $\mathrm{mmHg}(\Delta P)$

\begin{tabular}{rrrrrrrr}
\hline & $\mathbf{P I}$ & 200 & 180 & 160 & 140 & 120 & 100 \\
\hline & & & & & & & \\
\hline 10 & 34 & 35 & 35 & 37 & 40 & 40 \\
20 & 55 & 68 & 60 & 60 & 70 & 60 \\
30 & 80 & 85 & 90 & 85 & 90 & 75 \\
40 & 95 & 100 & 100 & 100 & 105 & 95 \\
50 & 110 & 120 & 110 & 115 & 115 & 105 \\
60 & 120 & 125 & 130 & 130 & 130 & 120 \\
70 & 136 & 145 & 140 & 140 & 140 & 140 \\
80 & 150 & 145 & 150 & 150 & 150 & 150 \\
90 & 160 & 155 & 164 & 160 & 160 & 160 \\
100 & 170 & 170 & 175 & 175 & 180 & - \\
110 & 180 & 180 & 180 & 185 & 185 & - \\
120 & 190 & 190 & 190 & 195 & - & - \\
130 & 200 & 195 & 200 & 200 & - & - \\
140 & 210 & 210 & 215 & - & - & - \\
150 & 220 & - & - & - & - & - \\
\hline
\end{tabular}

Em função destes valores, foram elaborados gráficos que demonstraram uma relação linear direta e semelhante entre os valores de fluxo e as variaçōes de pressão. Esta relação mostrou-se independente das pressōes iniciais, mas apenas dependente das variações pressóricas, o que é demonstrado pelo Gráfico 1, onde se encontram superpostos todos os gráficos obtidos para diferentes valores da pressão inicial. 


\section{FLUXO $\times$ VARIAÇÃO PRESSÃO}

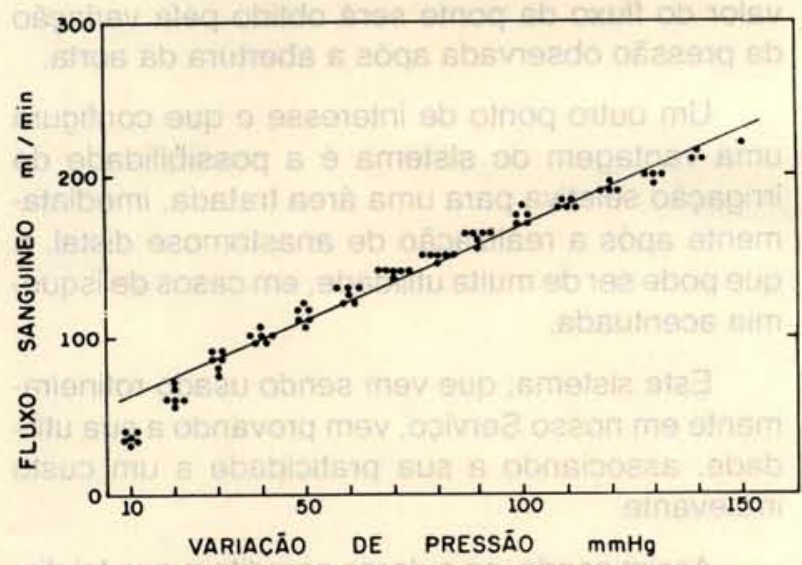

Gráf. 1 - Demonstrativo das variaçōes de pressão e fluxo sangüineo

\section{COMENTÁRIOS}

A avaliação dos fluxos dos enxertos aorto-coronários de veia safena tem sido de grande interesse, em muitos centros que se dedicam à cirurgia das coronárias. Isto se deve, sobretudo, à possibilidade, através do conhecimento dos fluxos avaliados durante $o$ ato operatório, de estabelecer previsões sobre o comportamento dos enxertos, principalmente com relação à sua perviabilidade a curto e longo prazos.

Esta avaliação é feita através do registro da magnitude e das características do fluxo. A magnitude é representada pelo fluxo médio do enxerto e está relacionada ao estudo da quantidade de sangue que se dirige á coronária, passando pela ponte, durante determinado tempo. Dentre as características do fluxo, estão incluídos o valor de suas medidas nos picos sistólico e diastólico, bem como seus valores durante a sístole e a diástole, e suas respectivas relações ${ }^{1,3.5}$.

Além disto, conforme MARCUS et alii ${ }^{4}$ (1981) e SEGADAL $^{9}$ (1985), o estudo da velocidade do sangue dentro do enxerto, durante a sístole e a diástole, parecem desempenhar um papel importante na perviabilidade dos enxertos a longo prazo 4. 9 . Sob o ponto de vista fisiológico, acredita-se que as condições ideais para que houvesse uma perviabilidade a longo prazo seriam a existência dentro do enxerto de alta velocidade central, baixa velocidade junto à parede do vaso e ausência de turbulência ${ }^{9}$.

Muitos métodos e dispositivos foram propostos e são utilizados para a avaliação destes fluxos. Basicamente, estes aparelhos, que são conhecidos por "fluxômetros", utilizam o efeito Doppler, ou eletromagnético, para realização das medidas ${ }^{7}, 8,9,10,11$.

São diversos os trabalhos que abordam este tema, relacionados ao aspecto técnico destas me- didas, ${ }^{2,}$, 7. 8, 10, 11 . Segundo SEGADAL ${ }^{9}$ (1985), estas medidas deveriam ser fáceis de realizar, fornecer resultados confiáveis, sobretudo em situaçōes de baixo fluxo, além de fornecer o maior número de informaçōes possíveis a respeito da quantidade e da qualidade do flux $0^{9}$. Embora os fluxômetros eletromagnéticos sejam os de uso mais difundido, sâo feitas algumas ressalvas quanto à sua utilizaçâo, sobretudo com relação ao seu sistema de calibração ${ }^{9}$. MORAN ${ }^{5}(1980)$ se reporta, especificamente, a este assunto, propondo, inclusive, um dispositivo para tornar este procedimento mais fácil ${ }^{6}$. Com relação aos aparelhos que utilizam o efeito Doppler, os resultados parecem muito satisfatórios, mesmo com diferentes métodos e equipamentos. Existem, já relatados, alguns estudos que visam à comparação entre os 2 métodos ${ }^{8} .9$. Entretanto, embora sejam dispositivos úteis, existem problemas que dificultam, ou impedem, a sua utilização. Como sâo aparelhos muito sensíveis, seu custo não é acessivel para a maioria dos Serviços, em nosso meio. Além disto, em função da sua própria constituição, são aparelhos que exigem manuseio e manutenção muito especializados que, normalmente, não estão disponiveis.

Em função destes aspectos e por considerar que a avaliação do fluxo das pontes é um bom indicador do prognóstico da cirurgia, os autores propōem o dispositivo em questão. Em termos gerais, é um dispositivo de funcionamento muito simples, prático, de custo irrelevante e que propicia a avaliação do fluxo das anastomoses com muita segurança. Em nosso Serviço, ele vem sendo utilizado rotineiramente. Ao término da anastomose distal, o ramo que se dirige ao campo operatório é acoplado à extremidade proximal e livre da veia safena, permitindo-se, a seguir, que o sangue do dispositivo flua para a veia. Como estão sendo avaliadas as pressões no dispositivo, antes e após a entrada do sangue no sistema, consegue-se obter a diferença entre estas pressões. Esta diferença representa uma quantidade de sangue que foi desviada para o sistema coronário, através da veia safena. Como a calibração do sistema é conhecida, consegue-se saber, com exatidão, o fluxo para o território coronário.

Alguns aspectos devem ser salientados. O primeiro é que os fluxos apresentados na Tabela 1 foram obtidos por calibração in vitro, estando o sangue com $28 \%$ de hematócrito. Além disto, os valores das medidas do fluxo são válidos quando a abertura lateral no conector metálico for $1 \mathrm{~mm}$ de diâmetro. Para alterações no hematócrito e no diâmetro, ocorrem alterações no fluxo. Optou-se por este calibre no ramo lateral por ele ser o que melhor propicia as medidas em função da unidade de tempo estabelecida em 1 minuto. $O$ segundo é que 
os valores de fluxo mostraram-se diretamente proporcionais à variação das pressōes prévia e posterior à abertura do ramo do campo operatório. Isto significa não haver relação entre as pressōes prévias na linha arterial e o fluxo obtido no enxerto, o que é devido à limitação de fluxo na saída lateral do conector.

O terceiro aspecto refere-se ao fluxo obtido após a abertura do ramo dirigido ao campo operatório. A quantidade de sangue que vai para o território coronário não vai representar o fluxo da ponte propriamente dito. Vai representar o fluxo total para o território coronário com a aorta fechada. No caso da artéria coronária estar ocluída proximalmente, o fluxo obtido será o mesmo da artéria coronária. Entretanto, em artérias que não apresentam obstrução total, haverá um fluxo natural pela coronária, além do da ponte. A soma destes 2 valores é que equivalerá ao fluxo total obtido pelo dispositivo. $O$ valor do fluxo da ponte será obtido pela variação da pressão observada após a abertura da aorta.

Um outro ponto de interesse e que configura uma vantagem do sistema é a possibilidade da irrigação seletiva para uma área tratada, imediatamente após a realização de anastomose distal, o que pode ser de muita utilidade, em casos de isquemia acentuada.

Este sistema, que vem sendo usado rotineiramente em nosso Serviço, vem provando a sua utilidade, associando a sua praticidade a um custo irrelevante.

Assim sendo, os autores acreditam que tal dispositivo, proposto para a medida do fluxo das pontes aorto-coronárias, seja útil, de fácil manuseio e custo desprezível.

RBCCV

JATENE, F. B.; LEMOS, P. C. P.; JATENE, A. D. - Hidro-dynamic flowmeter in myocardial revascularization surgery. Rev. Bras. Cir. Cardiovasc., 1 (1): 49-53, 1986.

ABSTRACT: Evaluation of blood flow in saphenous vein grafts during coronary surgery gives valuabie information about myocardial perfusion and long-term by- pass patency as well. However, electronic flowmeter availability is a limiting factor because of costs and maintenance. A system was developed consisting of a connection to the arterial line, with a $Y$ tube; one branch of the $Y$ tube is connected to a manometer and the other one to the vein graft. Since the connection to the arterial line has a know diameter, pressure drop in the system is proportional to the flow in the graft. With previous calibration, a chart was constructed that allows instantaneous evaluation of the flow. Another advantage of the system is the selective perfusion of the area to be treated before the conclusion of the proximal anastomosis. This system is being used in our Service; it is efficient and practical.

DESCRIPTORS: flowmeter, hidro-dynamic; myocardial revascularization.

\section{REFERÊNCIAS BIBLIOGRÁFICAS}

1 BALDERMAN, S. C.; MORAN, J. M.: SCANLON, P. J.; PIFARRE, R. - Predictors of late aorta-coronary graft patency: intra-operative phasic flow versus angiography. J. Thorac. Cardiovasc. Surg., 79: 724-728, 1980

2 FitzGERALD, D. E.; FORTESCUE-WEBB, C. M.; EKESTROM, S.; LILJEQVIST, L.; NORDHUS, O - Monitoring coronary artery blood flow by doppler shift ultrasound. Scand. J. Thorac. Cardiovasc. Surg., 11: 119-123, 1977.

3 FOLTS, J. D.; KAHN, D. R.; BITTAR, N.; ROWE, G. G - Effects of partial obstruction on phasic flow in aortocoronary grafts. Circulation, 51/52 (Supl. 1): 148-155, 1975.

4 MARCUS, M.; WRIGHT, C.; DOTY, D.; EASTHAM, C.; LAUGHLIN, D.; KRUMM, P.; FASTENOW, C.; BRODY, M. - Measurements of coronary velocity and reactive hyperemia in the coronary circulation of humans. Circ. Res., 49: 877-891, 1981.
5 MORAN, J. M.; CHEN, P. Y.; RHEINLANDER, H. F. Coronary hemodynamics following aorta-coronary bypass graft. Arch. Surg. . 103: 539-544, 1971.

6 MORAN, J. M.; BURKE, D. W.; LOEB, J. M.; ROBERTS, A. J.; SANDERS Jr., J. H.: MICHAELIS, L. L. - Accuracy in coronary graft fow measurement. Ann. Thorac. Surg., 32: 506-509, 1981.

7 MOULDER, P.V.; TEAGUE, M. J.; MANUELE, V. J.; BRUNSWICK, R. A.; DAICOFF, G. R. - Intraoperative doppler coronary artery finder. Ann. Thorac. Surg. 24:430-432, 1977.

8 RENEMAN, R. S.; CLARKE, H. S.; SIMMONS, N.; SPENCER, M. P. - In vivo comparison of electromagnetic and doppler flow-meters: with special attention to the processing of the analogue doppler flow signal. Cardiovasc. Res., 7: 557-566, 1973.

9 SEGADAL, L. - Assessment of flow in aortocoronary grafts. Semin. in Ultrasound, CT. and M.R., 6: 68-72, 1985. 
10 SEGADAL, L.; MATRE, K.; ENGEDAL, H.; RESCH, F. GRIP, A - Estimation of flow in aortocoronary graft with a pulsed ultrasound doppler meter. Thorac. Cardiovasc. Surg., 30: 265-268, 1982.

11 WRIGHT, C.; DOTY, D.; EASTHAM, C.; LAUGHLIN, D.; KRUMM, P.; MARCUS, M. - A method for assessing the physiologic significance of coronary obstructions in man at cardiac surgery. Circulation, 62 (Supl. 1). $111-115,1980$.

\section{Discussão}

DR. HÉLIO PEREIRA DE MAGALHÃES São Paulo, SP

A técnica de fluxometria por pressão diferencial acha-se descrita no livro Heart-lung bypass (Galetti, 1962), sendo primeiramente citada por Tosatti, em 1951. Revi os trabalhos: Cleland (1958) e Nixon (1959), onde referiram o uso da técnica para avaliar o fluxo extracorpóreo total; os 4 tipos de fluxometria por prersão diferencial são: 1) de resistência (usada pelos autores); 2 ) sistema Venturi; 3) de orifício e 4) tubo de Pitot. Faria algumas sugestões e observaçōes, quanto ao trabalho em questão: 1) substituir o termo "hidrodinâmico", pois não se trata de fluxo de água, para fluxômetro "hemodinâmico", ou mudar o título do trabalho para "Fluxometria transperfusional de ponte de safena por pressão diferencial"; 2) substituir o termo "perviabilidade" (forma irregular) por "permeabilidade", "permeação", ou "permeabilização"; 3) a quantificação do fluxo por pressão diferencial não exige conhecer o diâmetro de saida: este necessita ser menor; é determinado experimentalmente: não pode ser muito restritivo (determinando fluxos menores que o ensejado pela coronária) e não pode ser muito grande, acarretando excesso de fluxo e pressão a nível da anastomose, podendo ocorrer rotura na sutura, particularmente em vasos doentes; 4) a tabela por calibração prévia deve conter os valores de fluxos obtidos com toda a gama de pressão diferencial e a calibração deve ser feita com o sangue nas mesmas condições físicas de temperatura e hematócrito usadas durante a circulação extracorpórea; 5) o uso do sistema para irrigação seletiva, ou a própria irrigação, durante o período de medição, é vantajoso quando se usa parada anóxica hipotérmica intermitente, porém, quando se usa parada cardioplégica hipotérmica, a irriga- ção por essa técnica com sangue circulante de $28^{\circ}$ a $32^{\circ}$ vai criar gradientes de temperatura no miocárdio, o que poderá ser deletério para a proteção miocárdica; 6) considerar que, entre fluxometria trans e pós-perfusional, os fatores influentes são diversos: tipo de pulso (não pulsátil, ou pulsátil); temperatura (hipotermia, ou normotermia); viscosidade (maior, ou menor) e condições hemodinâmicas (dependência da bomba arterial, ou do ventrículo esquerdo). Na fluxometria transperfusional, considerar, também, a fuga de sangue pela lesão proximal da coronária, quando a aorta estiver aberta: lesōes obstrutivas menores deverão alterar os dados fluxométricos com valores acima do real. Como se trata de técnica prospectiva para a ponte de safena, seria interessante que os autores, para enriquecimento dessa pesquisa científica, cotejassem os valores fluxométricos obtidos por pressão diferencial transperfusional com valores obtidos por fluxometria eletromagnética pós-perfusional, para melhor avaliar a eficiência dos sistemas.

DR. F. JATENE ( Encerrando)

Gostaria, inicialmente, de agradecer o comentário do Dr. Hélio. Desejo salientar que alguns dos pontos e das questões abordadas também trouxeram dúvidas a nós, ou são detalhes que ainda estão em fase de ajuste. Um outro aspecto a ressaltar, e que nos parece muito claro, é que, quando do desenvolvimento e utilização do sistema, em nenhum momento tivemos a pretensão de desenvolver um sistema infalivel, e nem poderia ser desta forma. Temos plena consciência de que, mesmo os melhores fluxômetros eletrônicos apresentam problemas. Desta forma, o que não dizer de um fluxômetro simples, constituído por um conector metálico adaptado a alguns centímetros de tubos plásticos. O que eu gostaria que ficasse bem esclarecido é que nossa idéia, com este sistema, é a de ter sempre à mão e poder usar, em qualquer situação, um sistema simples, de custo irrelevante, fácil manuseio e, sobretudo, confiável, e que, esperamos, possa trazer benefícios aos Serviços que o utilizarem. Para finalizar, mais uma vez eu gostaria de agradecer a oportunidade da apresentação, bem como os comentários e as contribuições de todos. Muito obrigado. 